\title{
Молекулярные состояния композитных фермионов в самоорганизованных квантовых точках InP/GalnP в нулевом внешнем магнитном поле
}

\author{
(C) A.М. Минтаиров ${ }^{1,2}$ \\ ${ }^{1}$ Физико-технический институт им. А.Ф. Иоффе Российской академии наук, \\ 194021 Санкт-Петербург, Россия \\ ${ }^{2}$ Electrical Engineering Department, University of Notre Dame, \\ Notre Dame, Indiana 46556, USA \\ E-mail: amintairov@gmail.com
}

Поступила в Редакцию 26 сентября 2019 г.

В окончательной редакции 30 сентября 2019 г.

Принята к публикации 30 сентября 2019 г.

В одиночных квантовых точках $\operatorname{InP} / \mathrm{GaInP}$ с числом электронов $N=5-7$ и радиусом Вигнера-Зейтца $\sim 2.5$ определены размеры, положение областей локализаций линий и зависимость от магнитного поля $(0-10$ Тл) низкотемпературных $(10 \mathrm{~K})$ спектров фотолюминесценции с помощью ближнепольного сканирующего оптического микроскопа. Обнаружено формирование молекул композитных фермионов, имеющих размер областей локализации и длину связи $\sim 30$ и $\sim 50$ нм соответственно, для фактора заполнения уровней Ландау от $1 / 2$ до $2 / 7$ в нулевом внешнем магнитном поле. Для $N=6$ обнаружено спаривание и перестановка композитных ферментов, обусловленные фотовозбуждением, что открывает перспективы использования квантовых точек InP/GaInP для создания „немагнитного“ топологического квантового затвора.

Ключевые слова: квантовые точки InP/GaInP, композитные фермионы, фотолюминесценция, ближнепольная сканирующая микроскопия, топологический квантовый затвор.

DOI: 10.21883/FTP.2020.02.48893.9269

\section{1. Введение}

Самоорганизованные квантовые точки (KT) InP/GaInP, выращенные методом МОС-гидридной эпитаксии (газофазной эпитаксии из металлоорганических соединений), представляют собой естественные вигнеровские молекулы (ВМ), имеющие число электронов $(N)$ до 20 и радиус Вигнера-Зейтца $\left(r_{s}\right)$ до 5 [1-4]. В таких КТ может формироваться сильное встроенное магнитное поле, что обеспечивает режим квантового эффекта Холла [1] и возможность их использования для создания помехозащищенного топологического квантового затвора [5] без внешнего магнитного поля. При этом формирование BM в KT InP/GaInP может быть детально измерено с помощью фотолюминесценции (ФЛ) с высоким пространственным разрешением [4].

В процессе ФЛ ВМ с $N$ электронами (Ne-BM) при поглощении фотона заряженный $(N+1) e$ экситонный комплекс [6] не образуется, а происходит формирование $(N+1) e$-ВМ [3]. При этом квантовые состояния фотовозбужденной дырки премешиваются с квантовыми состояниями $N+1$ электронов за счет кулоновского взаимодействия. Это приводит к формированию $p$-, $d-, \ldots$ антистоксовых компонент (АСК) спектра ФЛ КТ, а релаксация электронов после излучения фотона индуцирует стоксовые компоненты (СК), обусловленные вращательно-колебательными модами $\mathrm{Ne}$-ВM [4]. При этом пространственное распределение интенсивности компонент спектра ФЛ определяется пространственным распределением электронной плотности $(N+1) e$-ВМ (для АCK) и $N e$-ВM (для СК), что позволяет измерять конфигурацию электронов в молекуле при использовании ближнепольного сканирующего оптического микроскопа (БСОМа) [4]. Такие измерения и их сопоставление с расчетами электронной плотности ВМ, выполненные методом конфигурационного взаимодействия, позволили наблюдать $2 e$-, $6 e-$ и $9 e$-ВМ с $r_{s} \sim 2.8-3.8$ в KT InP/GaInP и показать соответствие распределения электронной плотности этих ВМ классическим конфигурациям, т.е для $r_{s} \rightarrow \infty$ [7]. Эти измерения показали, что в некоторых КТ может наблюдаться существенное различие экспериментальных и расчетных распределений электронной плотности, обусловленное аномальной локализацией или делокализацией электронов. В настоящей работе мы определили размеры областей излучения и сдвиг линий спектра ФЛ в магнитном поле для ВМ с аномальной локализацией электронов (ВМЛ). Анализ экспериментальных данных показывает, что аномальная локализация обусловлена сильным встроенным магнитным полем ( 10 Тл), которое обеспечивает режим дробного квантового эффекта Холла, и формированием ВМ композитных фермионов (КФ) в нулевом внешнем магнитном поле. Полученные результаты открывают возможность использования KT InP/GaInP для создания „немагнитного“ помехоустойчивого топологического квантового затвора. 


\section{2. Детали эксперимента}

Исследуемый образец выращивался на точно ориентированной подложке $\mathrm{GaAs}(001)$ методом MOC-гидридной эпитаксии при температуре $700^{\circ} \mathrm{C}$ [8]. КТ формировали осаждением 8 монослоев InP на слой $\mathrm{Ga}_{0.52} \mathrm{In}_{0.48} \mathrm{P}$ толщиной 500 нм, согласованный по параметру решетки с $\mathrm{GaAs}$, и заращивали слоем $\mathrm{Ga}_{0.52} \mathrm{In}_{0.48} \mathrm{P}$ толщиной 40 нм.

Спектры ФЛ одиночных квантовых точек измерялись с помощью БСОМ, с использованием волоконнооптических зондов с Аl-покрытием и апертурами до 50 нм. Спектры измерялись при температуре $10 \mathrm{~K}$ и магнитных полях до 10 Тл, в режиме возбуждениесбор, с использованием многоканальной кремниевой линейки (CCD) и монохроматора с фокусным расстоянием 280 мм при возбуждении линией 514.5 нм Ar-лазера. Мощность возбуждения на входе в волокно составляла $\sim 5$ мкВт, что соответствует плотности возбуждения $\sim 0.5 \mathrm{BT} / \mathrm{cm}^{2}$.

Величины квантового ограничения $\hbar \omega_{0}$ и $N$ оценивались из величины расщепления и числа АСК. Пространственное распределение (карты) интенсивности для выбранной спектральной компоненты (пика) строилось с использованием спектров, измеренных при сканировании с шагом $50 \mathrm{Hм}$, и разложения спектра на лоренцевы компоненты. Область локализации пика излучения соответствовала области карты с интенсивностью 0.9 от максимума.

Размер КТ $D$ определяли из интегральной БСОМкарты. Радиус Вигнера-Зейтца рассчитывался по формуле $r_{s}=4 N / \pi D^{2} a_{\mathrm{B}}^{*}$, где $a_{\mathrm{B}}^{*}-$ боровский радиус; в эффективных атомных единицах $a_{\mathrm{B}}^{*}=\hbar^{2}\left(4 \pi \varepsilon \varepsilon_{0}\right) / m^{*} e^{2}$, где $\varepsilon_{0}, \varepsilon$ и $m^{*}$ - электрическая постоянная, диэлектрическая проницаемость и эффективная масса; для InP $a_{\mathrm{B}}^{*}=8.7 \mathrm{HM}$.

Величина встроенного магнитного поля $B_{\text {in }}$, эффективный заряд электрона $e^{*}$ и соответствующий фактор заполнения уровней Ландау $v_{0}=4 N h /\left(\pi D^{2} e_{0} B_{\text {in }}\right)$ оценивались по спектру Фока-Дарвина $E_{k, l}\left(B_{0}\right)=$ $=E_{k, l}\left(B+B_{\text {in }}\right)$, где $E_{k, l}\left(B_{0}\right)=E_{k, l}^{\mathrm{FD}}(\Omega), \Omega^{2}=\omega_{0}^{2}+\omega_{c}^{2 / 4}$, где $B$ - внешнее магнитное поле, $\omega_{c}^{*}=e^{*} B_{0} / m^{*}-$ циклотронная частота, $k(k=0,1, \ldots)$ и $l(l=-k$, $-k+2, \ldots, k-2)$ - главное и азимутальное квантовые числа [9]. Подробное описание методов измерений и расчетов электронной плотности приведено в [4].

\section{3. Экспериментальые данные}

\section{1. Спектры фотолюминесценции вигнеровских молекул с аномальной локализацией электронов}

На рис. 1 представлены спектры ФЛ девяти ВМЛ, обозначенных $\mathrm{WM}_{\mathrm{L}} 0, \ldots, \mathrm{WM}_{\mathrm{L}} 8$, и одной „нормальной“ ВМ (WM-P4, см. P4 КТ в [4]). Для ВМЛ в спектре наблюдаются основной пик, обозначенный 1 ,

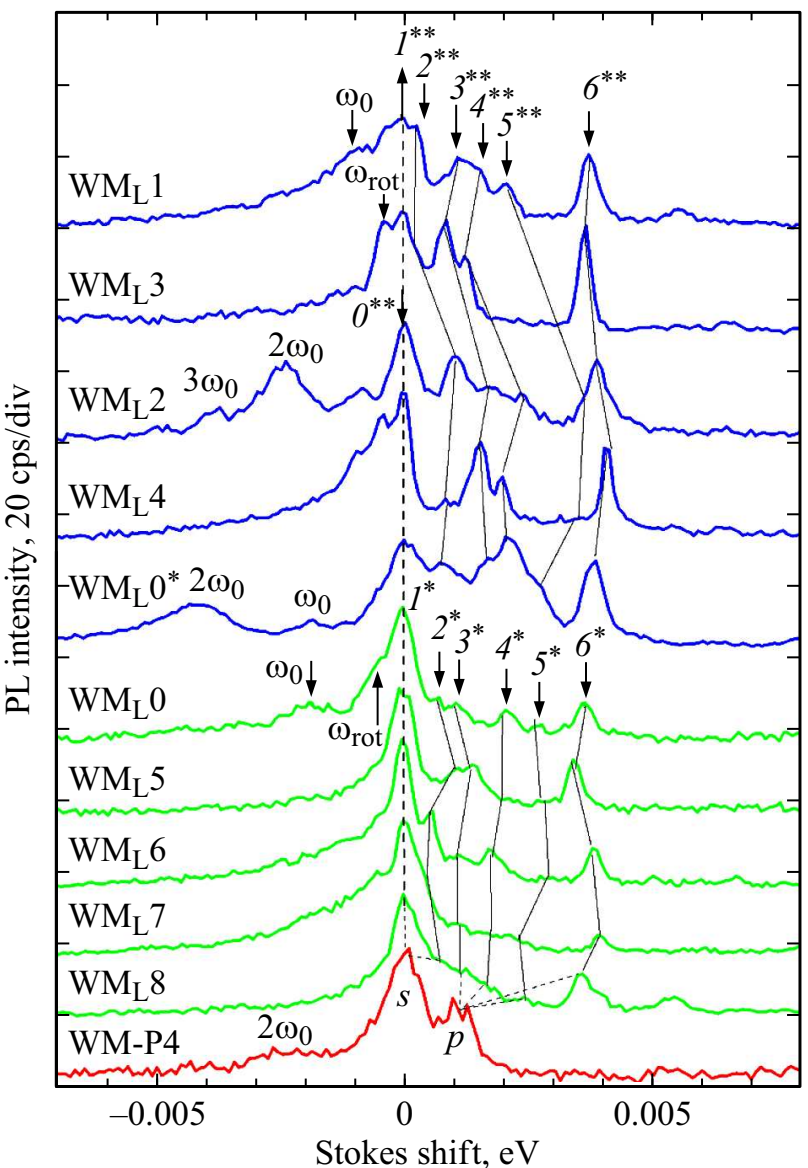

Рис. 1. Спектры фотолюминесценции (PL) (в шкале энергии стоксова сдвига) девяти „,аномальных“ и одной „нормальной“ $\mathrm{InP} / \mathrm{GaInP} \mathrm{BM}$. Спектр $\mathrm{BM} \mathrm{WM}_{\mathrm{L}} 0^{*}$ соответствует спектру $\mathrm{WM}_{\mathrm{L}} 0$ в магнитном поле 10 Тл.

до пяти АСК с энергией расщепления $\sim 1$ мэВ, обозначенных номерами $2-6$, а также вращательная $\left(\omega_{\text {rot }}\right)$ и трансляционная $\left(\omega_{0}\right)$ моды СК. Сравнение приведенных спектров показывает сильное различие в распределении интенсивностей пиков $1-6$ для $\mathrm{WM}_{\mathrm{L}} 0^{*}, \mathrm{WM}_{\mathrm{L}} 1-\mathrm{WM}_{\mathrm{L}} 4$, с одной стороны, и $\mathrm{WM}_{\mathrm{L}} 0, \mathrm{WM}_{\mathrm{L}} 5-\mathrm{WM}_{\mathrm{L}} 8-\mathrm{c}$ другой. Для первой серии ВМ интенивности АСК сравнимы с интенсивностью основного пика, а для второй они на порядок меньше. Чтобы различать эти два случая мы добавили индексы ${ }^{* *}$ и ${ }^{*}$ к номерам пиков соответственно. Для $\mathrm{WM}_{\mathrm{L}} 0$ в нулевом магнитном поле наблюдается распределение типа *, а при $B=10$ Тл - типа **. Кроме этих различий в спектрах ВМЛ с интенсивными АСК наблюдается более богатый и интенсивный спектр СК, включающий пики первого и второго обертонов трансляционной моды $\omega_{0}$.

Для нормальной ВМ в спектре наблюдаются один пик АСК, соответствующий $p$-оболочке, и один пик СК, соответствующий колебательной моде $\omega_{0}$. Для этой ВМ $N=5, \hbar \omega_{0}=1.1$ мэВ и $r_{s}=3.46$, а $s$ - и $p$-пики обусловлены рекомбинацией 2 и 4 электронов соответствующих вырожденных электронных состояний $6 e$-ВМ с дыркой. 

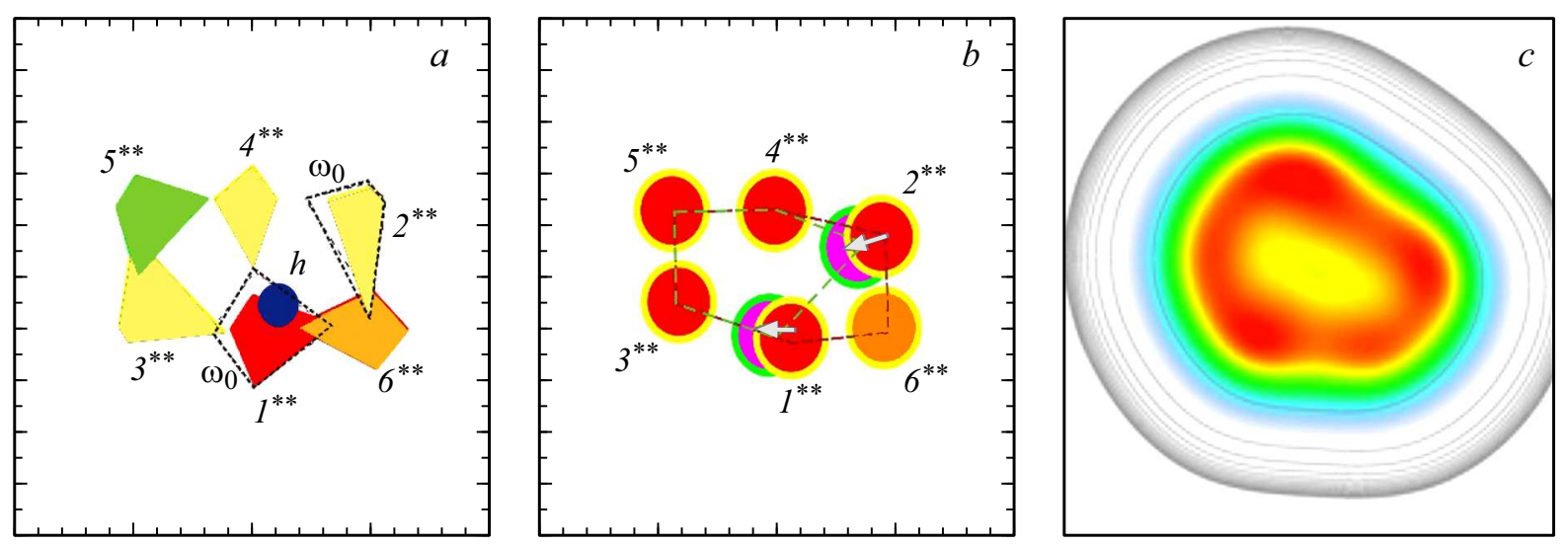

Рис. 2. Интегральная карта карты интенсивности пиков $1^{* *}-6^{* *}$ (сплошные фигуры) и $\omega_{0}$ (пунктирные фигуры) спектра ФЛ (a) и конфигурация $\mathrm{BM}$ в фотовозбужденном и исходном состояниях $\mathrm{WM}_{\mathrm{L}} 1(b)$ и расчетное распределение электронной плотности, соответствующей $6 e$-ВМ в нулевом магнитном поле [3] $(c)$. Цветной контраст $(a)$ соответствует уменьшению интенсивности в 3 раза, показано положение дырки $h$. Размер карт $200 \times 200$ нм $b$ : стрелки показывают смещение ОЛ пиков после рекомбинации.

Можно предположить, что в ВМЛ большое число АСК указывает на снятие вырождения и расщепление этих состояний, что в свою очередь указывает на спинполяризованное состояние электронов в КТ. Таким образом, пики $I^{*}-6^{*}$ и $I^{* *}-6^{* *}$ соответствуют рекомбинации одиночных электронов (пик $6^{*} / 6^{* *}$ соответствует фотовозбужденному электрону) с первого уровня Ландау, что предполагает их сильную локализацию и сильное встроенное магнитное поле, соответствующее $v_{0}<1$. Таким образом, можно заключить, что спектры ВМЛ на рис. 1 соответствуют $N \approx 5$ (в основном состоянии) и $N+1$ (в фотовозбужденном), $\hbar \omega_{0} \approx 2$ мэВ, $r_{s} \approx 2.5$ и $B_{\text {in }}>3$ Тл. При этом различие интенсивностей пиков $I^{*}-6^{*}$ и $I^{* *}-6^{* *}$ естественно связать с конфигурациями $(5,1)$ и $(6,0)$ изомеров $6 e$-ВМ или $(6,1)$ и $(5,0) 7 e$ - и $5 e$-ВМ соответственно. Для конфигурации $(5,1)$ и $(6,1)$ основной пик $\left(1^{*}\right)$, соответствующий рекомбинации центрального электрона и фотовозбужденной дырки, доминирует в спектре, в то время как для $(6,0)$ и $(5,0)$ все электроны равно удалены от дырки и соответсвующие пики рекомбинации имеют равную интенсивность. Согласно расчетам [10], состояние $(6,0)$ является основным для состояний, имеющих полный орбитальный момент $L=21,39$ и 51, которым для $\operatorname{InP}$ и $r_{s} \approx 2.5$ соответствуют $B \sim 4, \sim 7$ и $\sim 12$ Тл. Основные состояния изомера $(5,1)$ соответствуют $L=25,30,35$, $40,45,50$.

\section{2. Измерения области локализации пиков фотолюминесценции}

На рис. 2 и 3 показаны карты распределения интенсивности пиков спектра ФЛ и соответсвующие конфигурации $\mathrm{WM}_{\mathrm{L}} 1$ и $\mathrm{WM}_{\mathrm{L}} 2$, имеющих $N=5$ и 6 соответственно. Для пиков АСК размер области излучения составляет $l_{e} \approx 30$ нм с расстоянием между областями до $\sim 50$ нм. Для СК $l_{e} \approx 30-70 \mathrm{Hм}$.
Пространственная конфигурация областей локализации (ОЛ) пиков $1^{* *}-6^{* *}$ для $\mathrm{WM}_{\mathrm{L}} 1$ на рис. 2 образует вытянутый гексагон размером $70 \times 110$ нм. При этом интенсивность максимальна для пиков $1^{* *}, 6^{* *}$ и монотонно уменьшается для пиков $2^{* *}, 3^{* *}, 4^{* *}, 5^{* *}$, что соответствует их удалению от ОЛ дырки вблизи пика $l^{* *}$. Форма карты пика $\omega_{0}$ соответствует горизонтальному смещению ОЛ пиков $I^{* *}$ и $2^{* *}$ на $\sim 20$ нм после рекомбинации и описывает генерацию трансляционной моды в спектре ФЛ. Пространственная модуляция интенсивности пиков соответствует амплитуде модуляции электронной плотности $\mathrm{WM}_{\mathrm{L}} 1100 \%$, в то время как расчетная величина модуляции для нулевого магнитного поля, представленная на рис. 2, справа, составляет величину $\sim 15 \%$. В магнитном поле $l_{e}$ составляет величину $\sim 4 l_{B}=\left(\hbar / m^{*} \Omega\right)^{0.5}$, где $l_{B}$ - магнитная длина, и $l_{e}$, измеренная для $\mathrm{WM}_{\mathrm{L}} 1$, соответствует $B \sim 13$ Тл, $L=51$, что в свою очередь соответствует фазе дробного квантового эффекта Холла с $v_{0} \approx 2 / 7$. Эта фаза соответствует заполнению композитными фермионами (КФ) [11], состоящими из одного электрона и двух квантов магнитного потока $\left(К \Phi_{2}\right)$, нижнего уровня Ландау К $\Phi_{2}$, т. е. $v_{0 \text { CF }} \sim 1$. Таким образом, пики в спектре ФЛ соответствуют одиночным КФ 2 .

Для $\mathrm{WM}_{\mathrm{L}} 2 l_{e}$ пиков $2^{* *}, 3^{* *}$ и $4^{* *}$ (см. рис. 3 ) имеют такую же величину $\sim 30$ нм, как и для $\mathrm{WM}_{\mathrm{L}} 1$, что также соответствует $B \approx 13$ Тл и формированию КФ. Для этой $\mathrm{BM}$ пики $5^{* *}$ и $6^{* *}$ сливаются и область излучения пика $5^{* *}$ составляет суперпозицию ОЛ этих пиков (см. вставку к рис. $3, a)$. Кроме того, излучение пика $l^{* *}$ имеет две ОЛ, отстоящие друг от друга по диагонали влево вниз на $\sim 70$ нм, что соответствует дополнительному КФ $-O^{* *}$. При этом пространственная локализация пиков $1^{* *}, 2^{* *}, 3^{* *}$ и $6^{* *}$ совпадает в пределах $10 \mathrm{Hм}$, а ОЛ пиков $4^{* *}, 5^{* *}$ и $0^{* *}$ образуют равносторонний треугольник с базой 90 нм и длиной сторон 50 нм, отстоящий от ОЛ остальных пиков на 30 нм вниз по диагонали. 

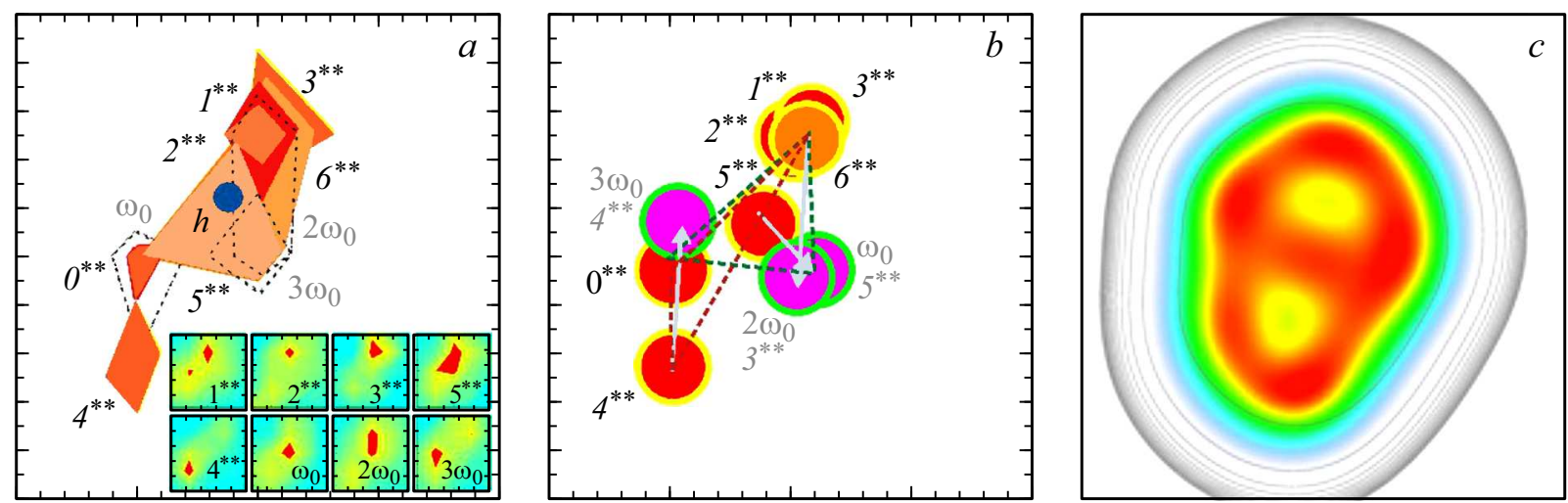

Рис. 3. Интегральная карта интенсивности пиков $1^{* *}-6^{* *}$ (сплошные фигуры) и $\omega_{0}, 2 \omega_{0}, 3 \omega_{0}$ (пунктирные фигуры) спектра ФЛ $(a)$, конфигурация ВМ в фотовозбужденном и исходном состояниях $\mathrm{WM}_{\mathrm{L}} 2(b)$ и расчетное распределение электронной плотности, соответствующей 7e-ВМ в нулевом магнитном поле [3] (c). (a): показано положение дырки $h$, на вставке - индивидуальные карты пиков, размер карт $200 \times 200$ нм. $b$ : стрелки показывают смещение ОЛ пиков после рекомбинации.

$a$

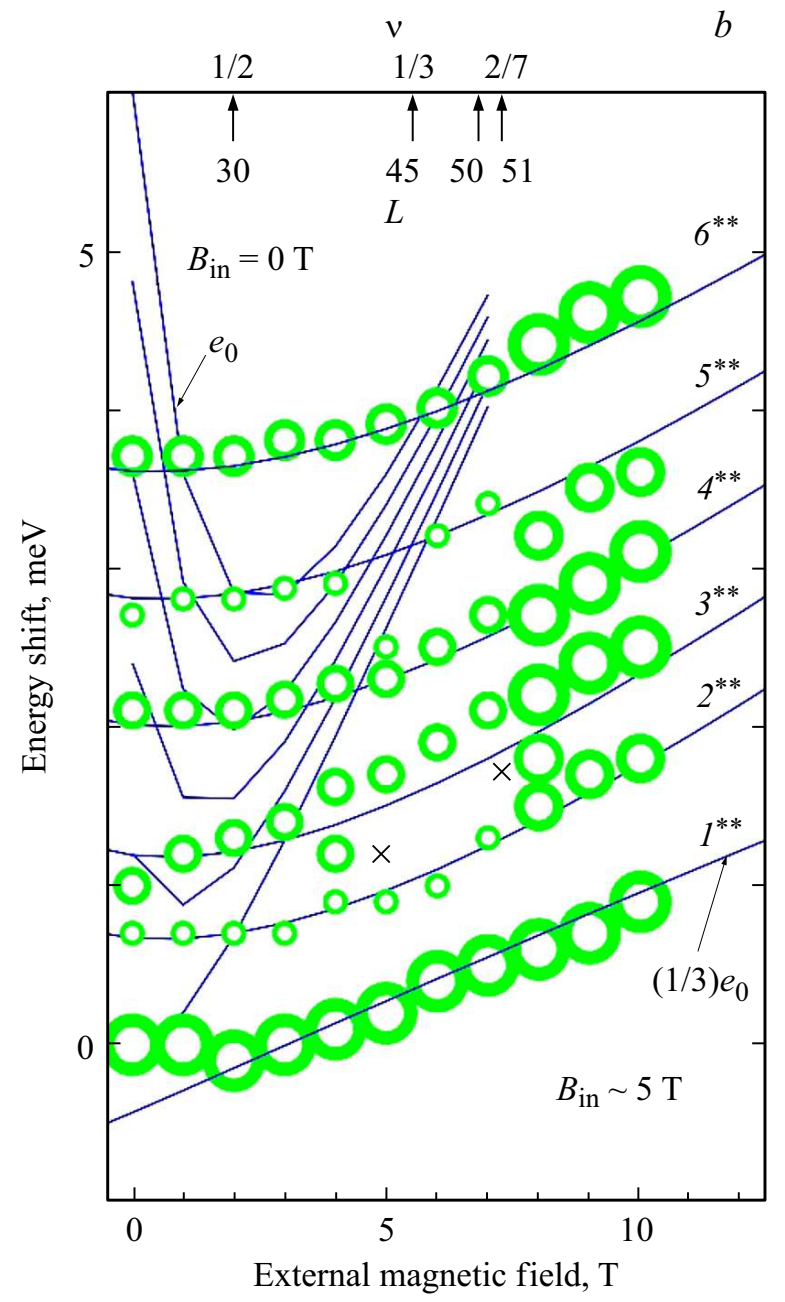

Рис. 4. Спектры ФЛ $\mathrm{WM}_{\mathrm{L}} 0$ в магнитных полях $0,2,4,6,8$ и 10 Тл $(a)$ и сдвиг линий пиков $1^{* *}-6^{* *}$ в зависимости от магнитного поля ( $b$, точки - эксперимент, линии - спектр Фока-Дарвина). $a$ : тонкие кривые - лоренцевы контуры, столбики в положениях максимумов - пиковые интенсивности линий; $b$ : размер кружков соответствует интенсивности пиков, стрелки у верхней оси показывают значения $v$ и $L$. 
Таким образом, постранственная конфигурация этой ВМ образует квазиодномерную структуру, представляющую собой сильно сплющенный треугольник с базой 120 нм, высотой 20 нм, имеющий на верхней вершине 4 спаренных КФ. При этом интенсивность пиков имеет почти одинаковую интенсивность, что соответствует локализации дырки вблизи центра треугольника. Анализ ОЛ пиков СК показывает, что пик $\omega_{0}$ соответствует смещению ОЛ пика $5^{* *}$ на 30 нм вправо по диагонали вниз, пик $2 \omega_{0}$ соответствует смещению ОЛ пика $3^{* *}$ на 50 нм вниз, а пик $3 \omega_{0}$ соответствует смещению ОЛ пика $4^{* *}$ на 50 нм вверх. Результирующая конфигурация ВМ представляет собой квазиравносторонний треугольник с длиной стороны $\sim 60$ нм и двумя спаренными КФ на каждой вершине. Наблюдаемые конфигурации КФ в основном и фотовозбужденном состояниях существенным образом отличаются от $\mathrm{WM}_{\mathrm{L}} 1$ и от ожидаемых $(6,0),(6,1)$ (см. рис. 3 , слева). При этом для $\mathrm{WM}_{\mathrm{L}} 2 v_{0} \approx 1 / 4-2 / 9$ и соответствует $К \Phi_{4}$, состоящим из электрона и четырех квантов магнитного потока.

\section{3. Спектры фотолюминесценции в магнитном поле}

На рис. 4, $a$ показаны спектры ФЛ $\mathrm{WM}_{\mathrm{L}} 0$ в магнитных полях $B=0,2,4,6,8$ и 10 Тл. Для каждого спектра приведены лоренцевы компоненты пиков, а столбиками (их высотой), расположенными на положениях максимумов, показана их интенсивность. При увеличении магнитного поля энергия максимума пиков увеличивается и для $B=10$ Тл сдвиг составляет $\sim 1$ мэВ. При этом для $B=4$ и 8 Тл в спектре появляется дополнительный пик, отстоящий на $\sim 0.3$ мэВ от пика $2^{* *}$, обозначенный $x$. Распределение интенстивностей пиков для $B=0$ и 2 Тл сооветствует $(5,1)$, а для 8 и 10 Тл - изомеру $(6,0)$ $6 e$-ВМ. Для $D=4$ и 6 Тл распределение интенсивностей пиков близко к распределению интенсивности для 0 и 2 Тл, но пики $3^{* *}$ и $6^{* *}$ имеют почти в 2 раза бо́льшую интенсивность. Заметим, что изменения в распределении интенсивностей пиков в магнитном поле происходит для $B=4$ и 8 Тл, для которых в спектре появляется пик $x$.

На рис. $4, b$ приведены сдвиги линий пиков $1^{*}-6^{*}$ в магнитном поле и сдвиги Фока-Дарвина (ФД). Видно, что спектр ФД описывает эксперимент для $B_{\text {in }} \approx 5$ Тл и $\omega_{c}^{*}=(1 / 3) \omega_{c 0}$, что соответствует заряду К $\Phi_{2}(1 / 3) e_{0}$, где $e_{0}$ - заряд электрона. На рисунке на вертикальной оси показаны значения $v=1 / 2,1 / 3$ и $2 / 7$, полученные с учетом встроенного поля, т.е. для значений магнитного

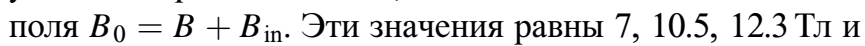
соответствуют $L=30,45,51$, что практически совпадает с расчетами для $6 e-\mathrm{BM}[10]$. Значение $v=1 / 2$ соответствует формированию жидкости/капли Ферми К $\Phi_{2}$, и, таким образом, наблюдаемые изменения распределения интенсивностей в спектре ФЛ соответствуют формированию изомеров $(5,1)$ и $(6,0) 6 e$-ВМ $К \Phi_{2}$ на первом уровне Ландау при $B_{0} \approx 9$ Тл для $v=1 / 2$ и $B_{0} \approx 12$ Тл для $v=2 / 7$. Появление пика $x$ можно связать с пересечением соседних состояний $L=30$ и $45, L=50$ и 51. Для $L=30,45,50$ и 51 набор одночастичных состояний включает в себя значения углового момента $l\{4,5,6,7,8,0\},\{5,6,7,8,9,10\}$, $\{5,7,8,9,10,11\}$ и $\{5,7,8,9,10,12\}$, и пик $x$ может соответствовать $l=10$ и 12.

\section{4. Обсуждение}

Наблюдение квазиодномерной молекулярной структуры $\mathrm{BM}$ для $\mathrm{WM}_{\mathrm{L}} 2$ крайне необычно, однако такая структура наблюдалась нами и для ВМ с меньшим $r_{s}$ $\left(r_{s} \approx 1.64\right)$ при измерениях интегральной формы ОЛ ВM, в которых отдельные электроны/КФ не были разрешены. Эти наблюдения были интерпретированы нами как формирование мод Фабри-Перо электронов. Данные рис. 3 позволяют связать формирование такой структуры не с модами Фабри-Перо, а со спариванием КФ. В случае $\mathrm{WM}_{\mathrm{L}} 2$ фотовозбужденное состояние содержит спаренные и неспаренные $К \Phi_{4}$ и, за счет разницы в зарядах они вытягиваются вдоль большего диаметpa КТ. Формирование пар К $\Phi_{4}$ в основном состоянии $\mathrm{WM}_{\mathrm{L}} 2$ неожиданно. Возможно, по аналогии с состоянием $v=5 / 2$, для которых пары КФ $\Phi_{4}$ образуются на втором уровне Ландау, пары КФ 4 могут образовываться и в области $v=1 / 4-2 / 9$, т. е. вблизи числа заполнения $v_{\mathrm{CF}} \approx 5 / 2$. Именно эти состояния, имеющие неабелеву квантовую статистику, можно использовать для создания помехоустойчивого топологического квантового затвоpa [12]. Таким образом, данные, полученные для $\mathrm{WM}_{\mathrm{L}} 2$, показывают принципиальную возможность использования InP/GaInP для создания такого затвора, основная идея которого состоит в использовании нескольких локализованных квазичастиц - энионов [13] с неабелиевой квантовой статистикой, их пар и возможности их перестановки и склеивания $[5,14]$. КФ представляют собой энионы, и данные на рис. 3 показывают перестановку КФ $\Phi_{4}$-энионов (для $0^{* *}$ и $4^{* *}, 5^{* *}$ и $3^{* *}$ ), их склеивание (для $0^{* *}$ и $4^{* *}, 5^{* *}$ и $3^{*}$ ), спаривание (для $0^{* *}$ и $4^{* *}, 5^{* *}$ и $3^{* *}, 1^{* *}$ и $2^{* *}$ ) и даже склеивание пар (для $1^{* *}, 2^{* *}$, $3^{* *}$ и $\left.6^{* *}\right)$. При этом для KT InP/GaInP квантовый затвор может быть „немагнитным“, т.е. работать в нулевом внешнем магнитном поле, а также, учитывая, что измерения на рис. 3 проводились при $10 \mathrm{~K}$, а эксперименты по измерению магнитопроводимости энионов проводятся при $0.006 \mathrm{~K}$ [12], и при более высоких температурах.

Следует также отметить, что нами были обнаружены КТ с $B_{\text {in }} \approx 2$ Тл [1], что дает возможность реализовать $v_{0} \approx 5 / 2$.

\section{5. Заключение}

С использованием ближнепольного сканирующего оптического микроскопа были измерены области локализации линий и зависимость спектров ФЛ от магнитного поля для „аномальных“ вигнеровских молекул 
KT InP/GaInP с числом электронов $5-7$ и радиусом Вигнера-Зейтца $\sim 2.5$. Показано, что в этих КТ аномальная локализация пиков излучения ( 30 нм) обусловлена встроенным магнитным полем (5-10 Тл), что приводит к формированию молекул композитных фермионов в нулевом внешнем магнитном поле с фактором заполнения уровней Ландау $v_{0}$ от $1 / 2$ до $1 / 5$. Обнаружено спаривание и перестановка композитных фермионов, обусловленные фотовобуждением, что открывает перспективы использования КT InP/GaInP для создания „немагнитного“ топологического квантового затвора.

\section{Благодарности}

Автор выражает благодарность Д. Винокурову за предоставление образца и А.С. Власову за помощь в проведении эксперимента.

\section{Финансирование работы}

Работа финансирована фондом РНФ, грант № 19-1900246.

\section{Конфликт интересов}

Авторы заявляют, что у них нет конфликта интересов.

\section{Список литературы}

[1] A.M. Mintairov, J.L. Merz, S. Blundell. In: Fingerprints in the Optical and Transport Properties of Quantum Dots (InTech ISBN 978-953-51-0648-7). (2012) p. 126.

[2] J. Kapaldo, S. Rouvimov, J.L. Merz, S. Oktyabrsky, S.A. Blundell, N. Bert, P. Brunkov, N.A. Kalyuzhnyy, S.A. Mintairov, S. Nekrasov, R. Saly, A.S. Vlasov, A.M. Mintairov. J. Phys. D, 49, 475301 (2016)

[3] A.M. Mintairov, J. Kapaldo, J.L. Merz, A.S. Vlasov, S.A. Blundell. Phys. Rev. B, 95, 115442 (2017).

[4] A.M. Mintairov, J. Kapaldo, J.L. Merz, S. Rouvimov, D.V. Lebedev, N.A. Kalyuzhnyy, S.A. Mintairov, K.G. Belyaev, M.V. Rakhlin, A.A. Toropov, P.N. Brunkov, A.S. Vlasov, Yu.M. Zadiranov, S.A. Blundell, A.M. Mozharov, I. Mukhin, M. Yakimov, S. Oktyabrsky, A.V. Shelaev, V.A. Bykov. Phys. Rev B, 97, 195443 (2018).

[5] C. Nayak, S.H. Simon, A. Stern, M. Freedman, S. Das Sarma. Rev. Mod. Phys., 80, 1083 (2008).

[6] M. Ediger, G. Bester, A. Badolato, P.M. Petroff, K. Karrai, A. Zunger, R.J. Warburton. Nature Phys., 3, 774 (2000).

[7] S.M. Reimann, M. Manninen. Rev. Mod. Phys., 74, 1283 (2002).

[8] D.A. Vinokurov, V.A. Kapitonov, O.V. Kovalenkov, D.A. Livshits, Z.N. Sokolova, I.S. Tarasov, Zh.I. Alferov. Semiconductors, 33, 788 (1999).

[9] L. Jacak, P. Hawrylak, A. Wojs. Quantum Dots (Springer, Berlin, 1998) p. 176.

[10] C. Yannouleas, U. Landman. Phys. Rev. B, 84, 165327 (2011).

[11] J.K. Jain, T. Kawamura. Europhys. Lett., 29, 321 (1995).
[12] H. Fu, P. Wang, P. Shan, L. Xiong, L.N. Pfeiffer, K. West, M.A. Kastner, X. Lin. PNAS, 113, 12386 (2016).

[13] F. Wilczek. Phys. Rev. Lett., 48, 1144 (1982).

[14] A.Y. Kitaev. Ann. Phys., 303, 2 (2003).

Редактор Л.В. Шаронова

\section{Molecular states of composite fermions in self-organized InP/GalnP quantum dots in zero magnetic field}

\author{
A.M. Mintairov ${ }^{\mathbf{1 , 2}}$ \\ ${ }^{1}$ loffe Institute, \\ 194021 St. Petersburg, Russia \\ 2 Electrical Engineering Department, \\ University of Notre Dame, \\ Notre Dame, Indiana 46556, USA
}

Abstract We used near-field scanning optical microscope to measure size and positions of lines and the magnetic field dependence $(0-10 \mathrm{~T})$ of low-temperature $(10 \mathrm{~K})$ photoluminescence spectra of single InP/GaInP quantum dots with the number of electrons $N=5-7$ and the Wigner-Seitz radius $\sim 2.5$. The formation of composite fermion molecules having the size and the bond length $\sim 30$ and $\sim 50 \mathrm{~nm}$, respectively, for the filling factor of the Landau levels from $1 / 2$ to $2 / 7$ in a zero external magnetic field has been found. For $N=6$, pairing and permutation of composite fermions due to photoexcitation were discovered, which opens up prospects for using $\mathrm{InP} / \mathrm{GaInP}$ quantum dots to create a „magnetic-field-free“ topological quantum gates. 